\title{
Analysis of Electromagnetic Force And Formability of Tube Electromagnetic Bulging Based On Convex Magnetic Field Shaper
}

\section{Weiye Wu}

China Three Gorges University https://orcid.org/0000-0002-4046-9255

\section{Li Qiu}

China Three Gorges University

\section{A. Abu-Siada}

Curtin University

\section{Chenglin Wang}

Materials branch of State Grid Chongqing Electric Power Company

Jinbo Jiang ( $\nabla$ jinbojiang@163.com )

China Three Gorges University

\section{Research Article}

Keywords: Tube fittings, Electromagnetic bulging, Magnetic field shaper, Uniformity, Wall thickness

Posted Date: November 2nd, 2021

DOI: https://doi.org/10.21203/rs.3.rs-1024163/v1

License: (c) (i) This work is licensed under a Creative Commons Attribution 4.0 International License. Read Full License 
Analysis of Electromagnetic Force and Formability of Tube Electromagnetic Bulging Based on Convex Magnetic Field Shaper

Weiye Wu ${ }^{a}$, Li Qiu ${ }^{a, b}$, A. ABU-SIADA ${ }^{c}$, Chenglin Wang ${ }^{d}$, Jinbo Jiang ${ }^{a, *}$ a.College of Electrical Engineering \& New Energy, China Three Gorges University, Yichang 443002, China.

b.Hubei Provincial Key Laboratory of Operation and Control of Cascaded Hydropower Station, China Three Gorges University, Yichang 443002, China. c.Discipline of Electrical and Computer Engineering, Curtin University, Perth, WA 6102, Australia.

d.Materials branch of State Grid Chongqing Electric Power Company, Chongqing 401121, China.

Correspondence information: Jinbo Jiang, College of Electrical Engineering \& New Energy, China Three Gorges. Yichang 443002, China.

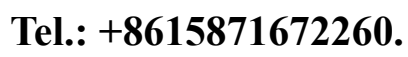

Email address: jinbojiang@163.com

ORCID: 0000-0003-1752-5877 


\title{
Analysis of Electromagnetic Force and Formability of Tube Electromagnetic
}

\section{Bulging Based on Convex Magnetic Field Shaper}

\author{
Weiye $\mathrm{Wu}^{\mathrm{a}}$, Li Qiu ${ }^{\mathrm{a}, \mathrm{b}}$, A. ABU-SIADA ${ }^{\mathrm{c}}$, Chenglin Wang ${ }^{\mathrm{d}}$, Jinbo Jiang ${ }^{\mathrm{a}, *}$
}

a.College of Electrical Engineering \& New Energy, China Three Gorges University, Yichang 443002, China.

b.Hubei Provincial Key Laboratory of Operation and Control of Cascaded Hydropower Station, China Three Gorges University, Yichang 443002, China. c.Discipline of Electrical and Computer Engineering, Curtin University, Perth, WA 6102, Australia.

d.Materials branch of State Grid Chongqing Electric Power Company, Chongqing 401121, China.

\begin{abstract}
In order to solve the problems of non-uniform axial deformation and thinning of wall thickness in traditional tube electromagnetic bulging, a method of tube electromagnetic bulging based on convex magnetic field shaper is proposed in this paper. The electromagnetic-structure coupling model is constructed by using COMSOL software, and the influence of convex magnetic field shaper structure on radial and axial electromagnetic force, axial deformation uniformity and wall thickness reduction is
\end{abstract}


Analysis of Electromagnetic Force and Formability of Tube Electromagnetic Bulging Based on Convex Magnetic Field Shaper

analyzed, and compared with traditional tube electromagnetic bulging. The results show that by using this method, the axial deformation uniformity is increased by 4.2 times, and the relative wall thickness is reduced by $33 \%$. Obviously, this method of tube bulging can effectively overcome the problems existing in traditional tube electromagnetic bulging and promote the wide application of electromagnetic forming technology.

Keywords: Tube fittings; Electromagnetic bulging; Magnetic field shaper; Uniformity; Wall thickness 


\section{Introduce}

Electromagnetic forming is a kind of high-speed machining technology which uses the Lorentz force on the induced current on the workpiece to drive the forming of the part [1-3]. Electromagnetic forming is environmentally friendly and energy-saving, and it is easy to realize automatic mass production, and many metals have high formability under the condition of electromagnetic forming, which expands the application range of high strength and low formability metals. It is widely used in aerospace, electronics, automobile and other industrial fields.

According to the workpiece shape, electromagnetic forming can be divided into tube electromagnetic forming and plate electromagnetic forming. The tube electromagnetic forming can be divided into tube electromagnetic bulging and tube electromagnetic compression according to the way of electromagnetic force. However, there are some problems such as uneven axial deformation and thinning of tube wall after electromagnetic bulging.

Mamalis et al. [4] adopted the optimization analysis method of finite element analysis and numerical simulation by changing various parameters to better analyze the electromagnetic forming process. In [5], based on the optimization method of sequential coupling numerical simulation and experimental design, it is found that the uniform axial deformation trend of aluminum tube in the process of electromagnetic compression depends on the length ratio of tube to coil. The effects of coil length and relative position on the force and electromagnetic bulging forming properties of tubes are discussed in reference [6]. In [7], by using the electromagnetic progressive forming 
technology in which small coils discharge sequentially at different positions of the tube, the axial deformation of the tube is more uniform. In order to overcome the problem of uneven axial bulging caused by the end effect, Qiu et al. [8] designed a concave drive coil to reduce the radial electromagnetic force in the middle of the tube and improve the axial deformation uniformity of the tube.

In order to increase the radial flow of the plate and reduce the thickness reduction of the plate, an axial-radial bidirectional loading method is proposed in reference [9]. In [10], when the length of the tube is less than the length of the coil, the inhomogeneity and wall thickness of axial bulging can be reduced by changing the ratio of length to inner diameter and applying different electromagnetic forces. In [11], the three-coil is used to apply both radial force and axial force in the electromagnetic bulging of the tube to improve the axial uniformity of the tube and restrain the thinning of the wall.

A three-dimensional model of electromagnetic field distribution in electromagnetic field forming system is given in reference [12]. The results show that the magnetic field shaper not only enhances the magnetic field, but also uniformly distributes the magnetic field along the workpiece. Through the simulation calculation in [13], the magnetic field shaper can replace the three-dimensional model with two-dimensional axisymmetric model, and the simulation error is very small.

The electromagnetic force distribution of the tube can be changed by changing the number and shape of the coil and the position relationship between the coil and the tube fittings. However, the change of these parameters requires high requirements for the coil tooling in the actual production and is not easy to operate, and the economic benefit 
is poor. In this paper, a convex magnetic field shaper is introduced into the tube bulging system, which weakens the radial electromagnetic force in the middle of the tube and improves the uniformity of the axial deformation of the tube, and the outer wall of the magnetic field shaper is higher than that of the tube, which can strengthen the axial electromagnetic force on the tube. Promote the axial flow of tube materials and limit the thinning of tube wall thickness.

\section{Basic Principles}

The traditional electromagnetic bulging system consists of a charging system, a discharge capacitor, a switch, a drive coil, a tube fitting and a continuous current circuit. As shown in Fig 1 (a), the charging system first charges the capacitor, then closes the switch, and the energy stored by the capacitor is transferred to the drive coil in the form of a pulse current. The magnetic field generated by the coil itself is superimposed with the induced eddy current generated on the tube fitting to produce the electromagnetic force that drives the tube bulging.

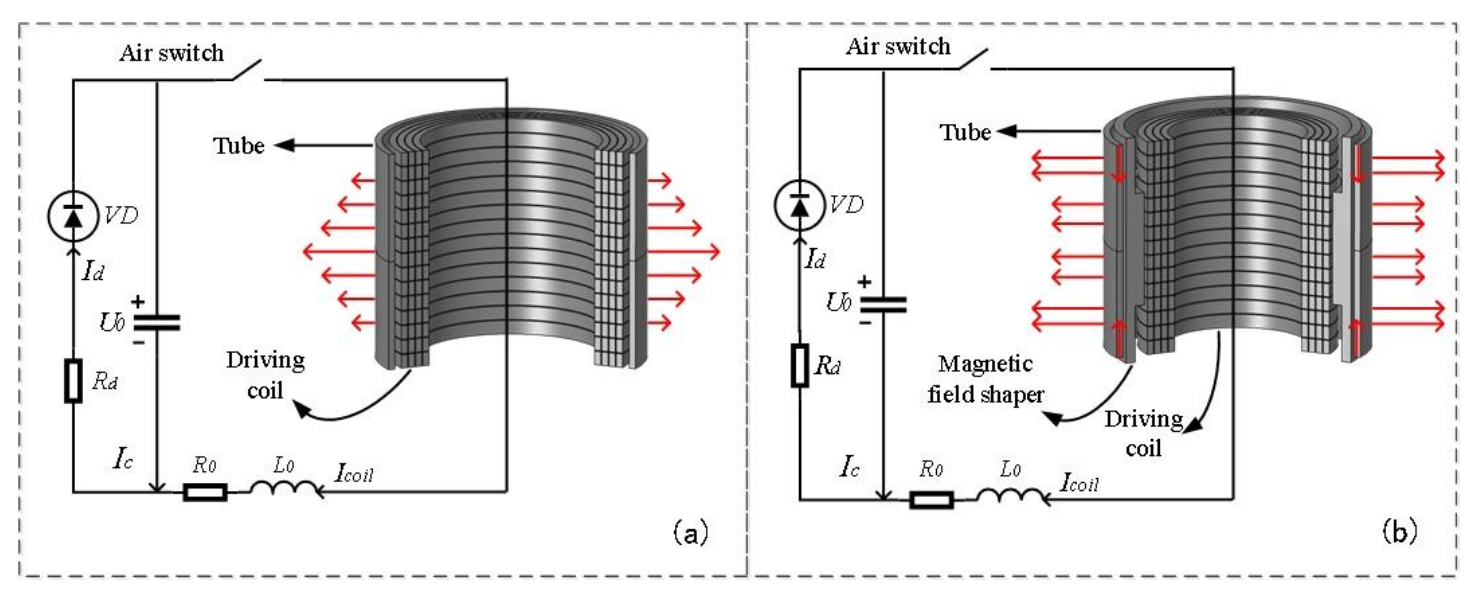

Fig.1 Electromagnetic bulging structure diagram (a) Traditional model; (b) Loading model based on convex magnetic field shaper. 
Analysis of Electromagnetic Force and Formability of Tube Electromagnetic Bulging Based on Convex Magnetic Field Shaper

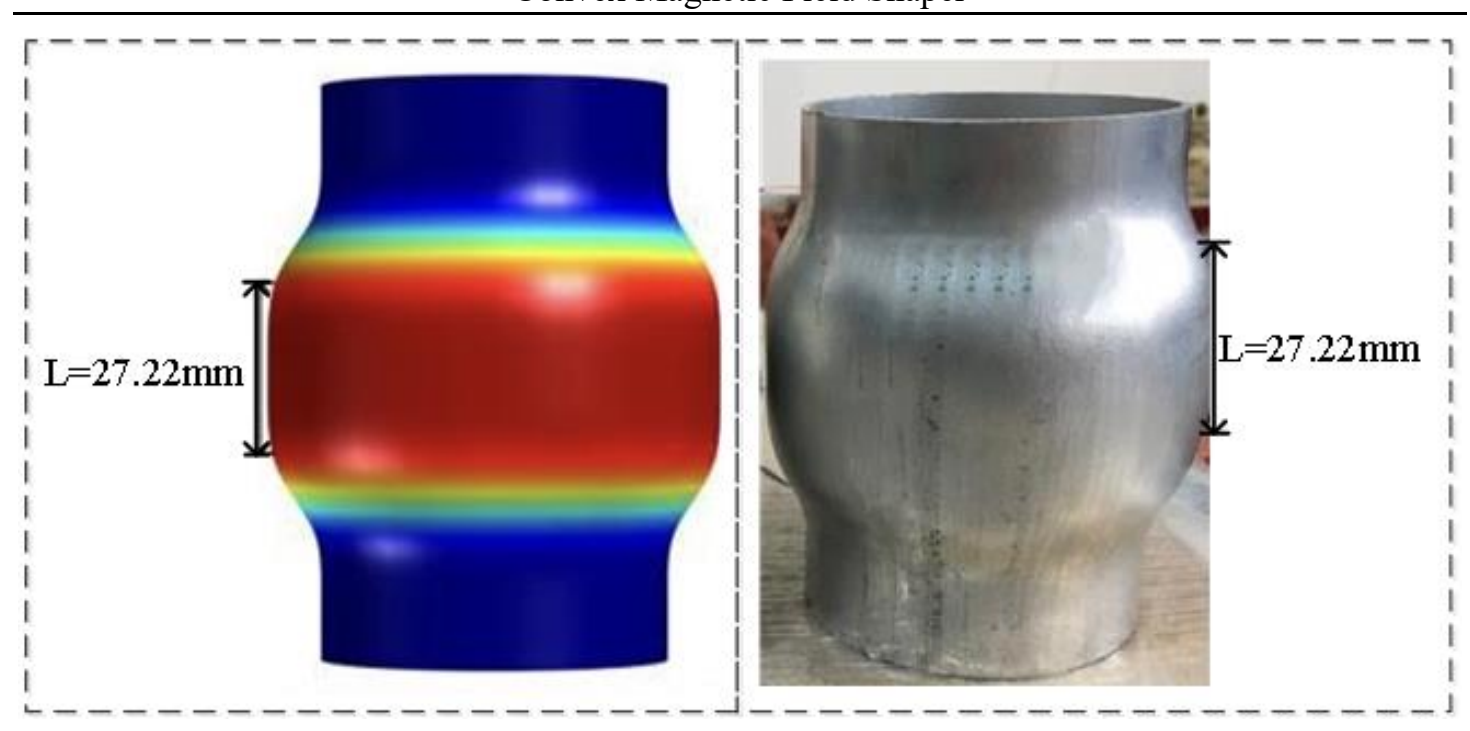

Fig.2 Electromagnetic bulging diagram (a) Simulation; (b) Experiment [14].

However, in the traditional coil tube electromagnetic bulging system, because the height of the driving coil is usually less than or equal to the height of the tube, the axial magnetic flux density is much larger than the radial magnetic flux density, so that the tube is subjected to a larger radial electromagnetic force, resulting in a large reduction of wall thickness. And the radial electromagnetic force is not uniformly distributed in the axial direction, which leads to the bulging of the tube into an axially uneven convex shape.

Through the experimental verification in [14], the electromagnetic bulging of the tube is loaded with a concave coil to weaken the radial electromagnetic force in the middle of the tube, which can make the axial deformation of the tube uniform. The experimental results are shown in Fig 2.

This article introduces a convex magnetic field shaper in the middle of the fittings and coils as shown in Fig 1 (b), after discharge of capacitance, the driving coil generates a pulsed strong magnetic field, and the induced current in the convex magnetic field shaper and fittings [15]. Where the convex magnetic field shaper inner wall length is 
smaller than the outer wall length, the inner wall current density is larger than the outer wall current density because the magnetic field shaper inner and outer wall currents are equal. The inner wall current forms a loop through the scission of the magnetic field shaper, so the direction of the inner wall current and the direction of the outer wall current are opposite [13]. Thus when the inductive vortex on the fitting interacts with the magnetic field resulting from the magnetic field shaper's induced current, the inner wall induced current creates a repulsion opposite to the direction of the outer wall induced current, weakening the radial electromagnetic force experienced in the middle of the fitting and increasing the uniformity of the fitting's axial deformation. Also because the convex magnetic field shaper outer wall length is greater than that of the fitting, it produces a greater than Radial flux, a traditional loading method, renders the fitting subject to large axial electromagnetic forces, promotes axial flow of material into the fitting, and inhibits the thinning of the fitting wall thickness.

\section{Numerical simulation}

The electromagnetic bulging model of two-dimensional axisymmetric tube is constructed by COMSOL, and the geometric structure of the model is shown in Fig 2. 


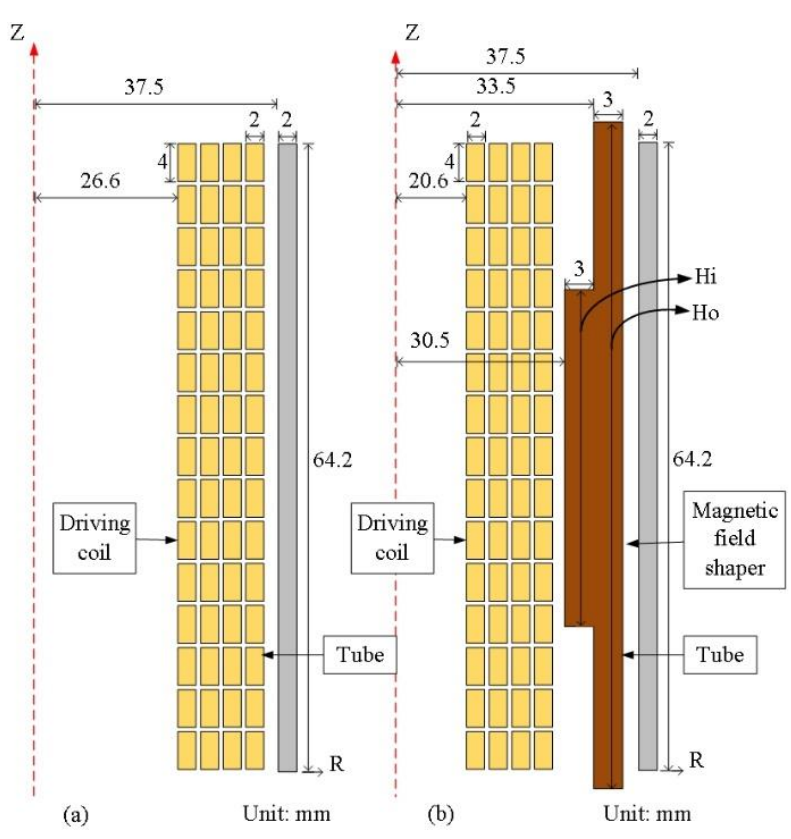

Fig.3 Geometrical structure diagram (a) Bulging of traditional tube fittings; (b)

Bulging of tube fittings loaded by convex magnetic field shaper.

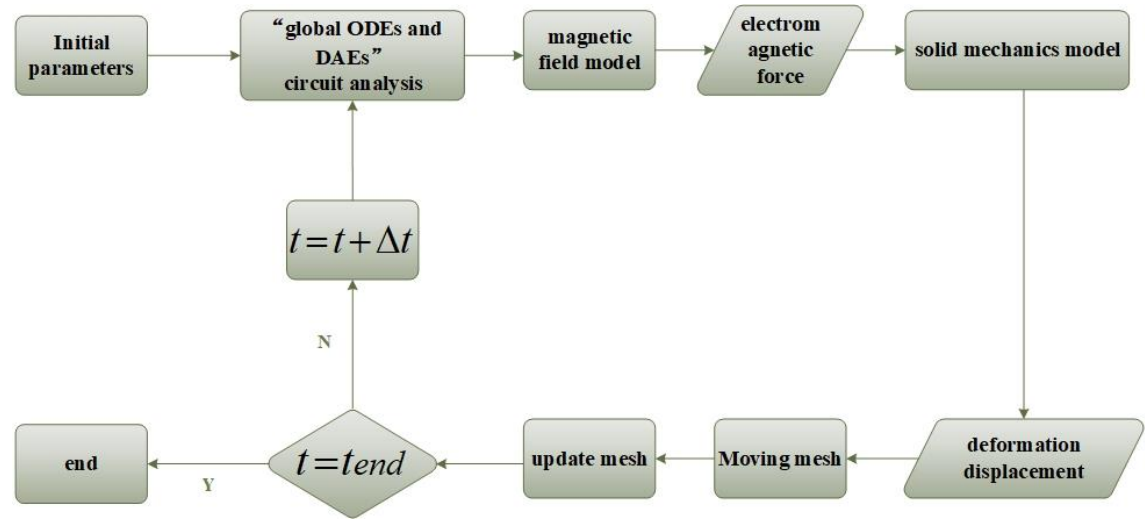

Fig.4 Bulging simulation flow chart of tube fittings.

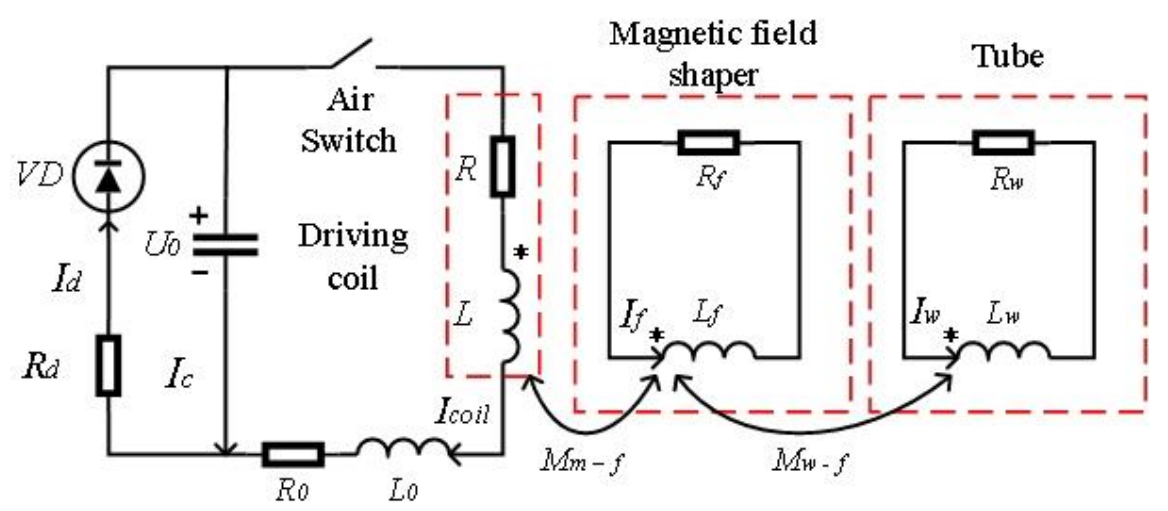

Fig.5 Electromagnetic bulging equivalent circuit of tube fitting based on convex 
magnetic field shaper.

Figure 4 shows the flow chart of electromagnetic bulging of tube fittings. The global ordinary differential equation and differential-algebraic equation module are used to solve and analyze external circuits. When there is a convex magnetic field shaper in the circuit, if the coupling between the coil and the tube fitting is ignored, the equivalent circuit of the tube electromagnetic bulging is shown in figure 5. The specific parameters of external circuits and fittings are shown in Table 1. According to Kirchhoff's law, the following equations are obtained:

$$
\left\{\begin{array}{l}
\left(R_{0}+R\right) I_{c o i l}+\left(L_{0}+L\right) \frac{\mathrm{d} I_{\text {coil }}}{\mathrm{d} t}=\boldsymbol{U}_{\boldsymbol{C}} \\
R_{W} \boldsymbol{I}_{W}+L_{W} \frac{\mathrm{d} \boldsymbol{I}_{W}}{\mathrm{~d} t}=0 \\
R_{f} \boldsymbol{I}_{f}+\boldsymbol{L}_{f} \frac{\mathrm{d} \boldsymbol{I}_{f}}{\mathrm{~d} t}=0 \\
\boldsymbol{U}_{\boldsymbol{C}}=\boldsymbol{U}_{\boldsymbol{o}}-\frac{1}{C} \int_{0}^{\mathrm{t}} \boldsymbol{I}_{C} d t
\end{array}\right.
$$

Among them, $U_{c}$ is the capacitive voltage; $I_{c}$ is the drive coil current; $I_{w}$ is the induced eddy current in the tube; $I_{f}$ is the induced current in the magnetic field shaper; $R$ is the drive coil resistance and $L$ is the drive coil inductance.

Table 2 External circuit and fitting parameters.

\begin{tabular}{ccc}
\hline Symbol & Meaning & Value \\
\hline 1. Circuit parameters & & \\
$C$ & The discharge Capacitance & $320 \mathrm{uF}$ \\
$L_{0}$ & Line inductance & $6.3 \mathrm{uH}$ \\
$R_{e}$ & Line resistance & $28 \mathrm{~m} \Omega$ \\
$R_{d}$ & Continuous resistance & $200 \mathrm{~m} \Omega$ \\
$U_{0}$ & Discharge voltage & $4.8 \mathrm{kV}$
\end{tabular}

2. Fitting parameters

$D_{i}$

Internal diameter

$75 \mathrm{~mm}$ 


\begin{tabular}{ccc}
$D_{o}$ & External diameter & $79 \mathrm{~mm}$ \\
$H_{t}$ & Height & $64.2 \mathrm{~mm}$ \\
$\rho$ & Density & $2700 \mathrm{~kg} / \mathrm{m} 3$ \\
$\gamma$ & Conductivity & $3.03 \mathrm{e}^{7} \mathrm{~S} / \mathrm{m}$ \\
$\sigma_{y s}$ & Initial yield stress & $55 \mathrm{MPa}$ \\
$\mu$ & Poisson's ratio & 0.33 \\
$E$ & Young's modulus & $70 \mathrm{e}^{9} \mathrm{~Pa}$ \\
\hline
\end{tabular}

The magnetic field module calculates the magnetic field intensity and the electromagnetic force on the tube, and introduces the electromagnetic force into the solid mechanics module. The solid mechanics module transfers the calculated force deformation of the tube to the magnetic field module to realize the electromagneticstructure coupling. The relationship between the electromagnetic force and the displacement of the tube fitting is as follows:

$$
\rho \frac{\partial^{2} \boldsymbol{B}_{z}}{\partial \boldsymbol{t}^{2}}-\nabla \times \sigma=\boldsymbol{F}
$$

Where $\sigma$ is the stress tensor of the tube, $\boldsymbol{F}$ is the volume density vector of the electromagnetic force, and $\boldsymbol{u}$ is the displacement vector of the tube." The Lorentz force is decomposed into axial Lorentz force $\left(\boldsymbol{F}_{z}\right)$ and axial Lorentz force $\left(\boldsymbol{F}_{r}\right)$, the flux density is decomposed into axial flux $\left(\boldsymbol{B}_{z}\right)$ and radial flux $\left(\boldsymbol{B}_{r}\right), \boldsymbol{J}_{\varphi}$ represents circumferential eddy current, and the Lorentz force is decomposed as follows:

$$
\begin{aligned}
& \boldsymbol{F}_{z}=\boldsymbol{J}_{\varphi} \times \boldsymbol{B}_{\boldsymbol{r}} \\
& \boldsymbol{F}_{\boldsymbol{r}}=\boldsymbol{J}_{\varphi} \times \boldsymbol{B}_{z}
\end{aligned}
$$

The constitutive equation of AA6061-O aluminum alloy tube used in this model is:

$$
\sigma=\left[1+\left(\frac{\varepsilon_{p e}}{C_{m}}\right)^{m}\right] \sigma_{y s}
$$

Where $m$ is the strain rate hardening parameter, $C_{m}$ is the viscous parameter, 
usually $C_{m}=6500, \quad m=0.25[16]$.

4 Formability analysis of branch tube based on load of convex magnetic field shaper

\subsection{Formability analysis of branch tube based on load of convex magnetic field} shaper

\subsubsection{Influence of $\mathrm{H}_{\mathrm{i}}$}

The inner wall height $\left(\mathrm{H}_{\mathrm{i}}\right)$ and outer wall height $\left(\mathrm{H}_{\mathrm{o}}\right)$ of the convex magnetic shaper were changed while the coil, tube fitting and other circuit parameters were kept unchanged, and the influence of the force distribution and magnitude of the tube fitting on the uniform axial deformation and wall thinning of the tube fitting during the loading of the convex magnetic shaper was analyzed. $\mathrm{H}_{\mathrm{i}}$ and $\mathrm{H}_{\mathrm{o}}$ were changed respectively within a certain range, and other parameters were kept unchanged. The range of $\mathrm{H}_{\mathrm{i}}$ was $8 \mathrm{~mm}-26 \mathrm{~mm}$ and the step length was $3 \mathrm{~mm}$, and the range of $\mathrm{H}_{\mathrm{o}} \quad$ was $28 \mathrm{~mm}-$ $40 \mathrm{~mm}$ and the step length was $2 \mathrm{~mm}$.

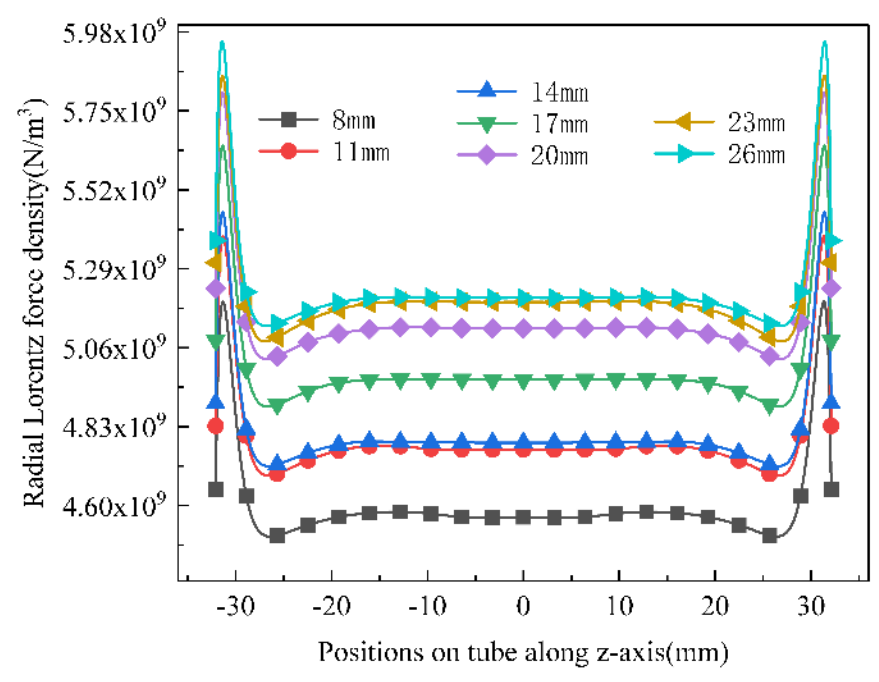

Fig.6 Change the radial electromagnetic force distribution of $\mathrm{H}_{\mathrm{i}}$

Fig 7 shows the influence of convex magnetic shaper inner wall height $\left(\mathrm{H}_{\mathrm{i}}\right)$ on the 
axial deformation of the tube fitting. As can be seen from the above electromagnetic force analysis, with the increase of $\mathrm{H}_{\mathrm{i}}$, the electromagnetic force subjected to the tube fitting changes from concave to convex at $-10 \mathrm{~mm}$ to $10 \mathrm{~mm}$, so the axial deformation of the middle part of the tube fitting changes from concave to convex, and there is an optimal $\mathrm{H}_{\mathrm{i}}=17 \mathrm{~mm}$, which makes the axial deformation of the middle part of the tube fitting uniform.

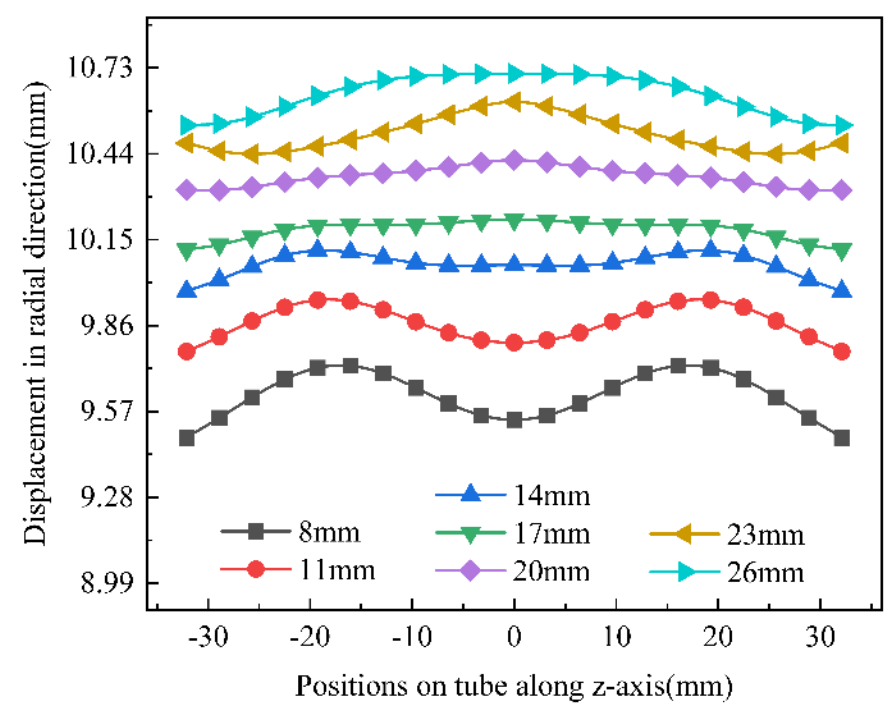

Fig.7 Change $\mathrm{H}_{\mathrm{i}}$ tube fitting axial deformation.

\subsubsection{Influence of $\mathrm{H}_{\mathrm{o}}$}

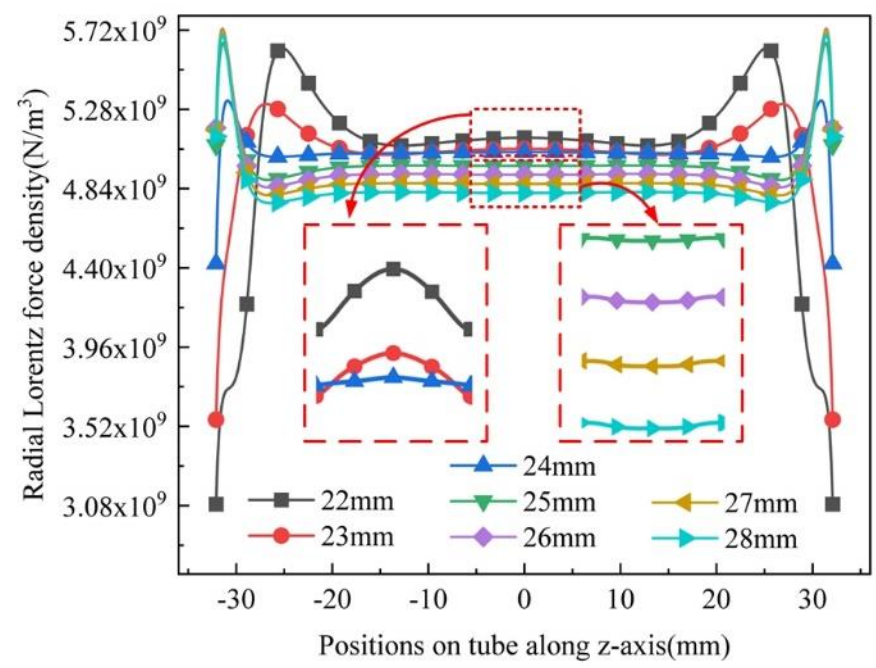


Fig.8 Change the radial electromagnetic force distribution of $\mathrm{H}_{\mathrm{o}}$.

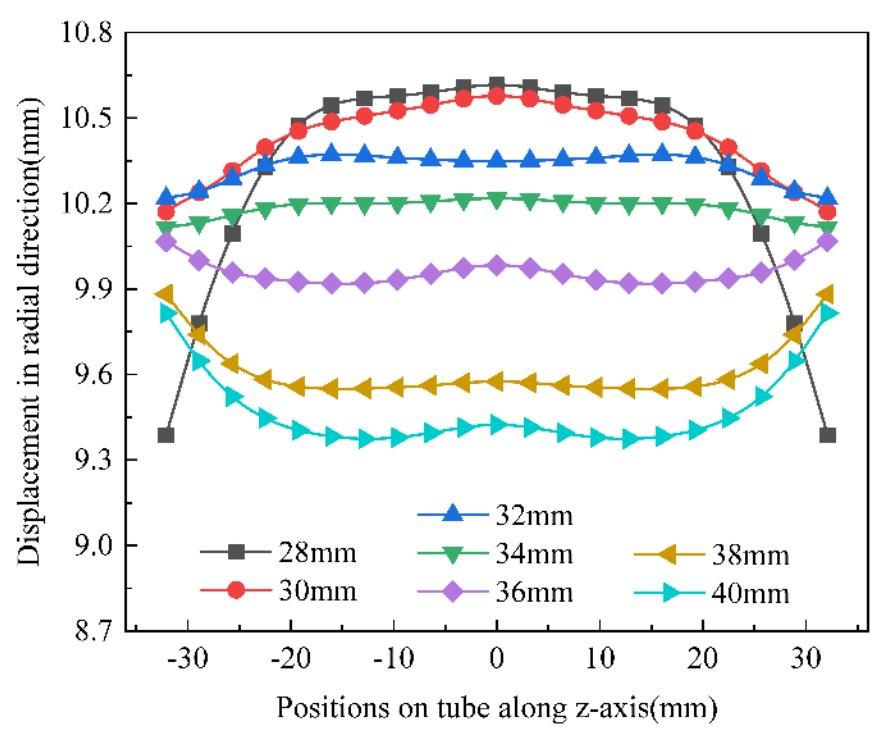

Fig.9 Change $\mathrm{H}_{\mathrm{o}}$ tube fitting axial deformation.

Fig. 8 shows the influence of the outer wall height $\left(\mathrm{H}_{\mathrm{o}}\right)$ of the convex magnetic shaper on the radial electromagnetic force. The radial electromagnetic force has a concave distribution on the tube fitting, and the electromagnetic force is evenly distributed in the middle part of the tube fitting. With the increase of $\mathrm{H}_{\mathrm{o}}$, the peak value of the radial electromagnetic force moves towards the middle of the tube fitting and deforms from concave to convex at $-10 \mathrm{~mm}-10 \mathrm{~mm}$. Therefore, changing the size of $\mathrm{H}_{\mathrm{o}}$ can change the size and distribution of radial electromagnetic force. Fig. 9 shows the influence of the outer wall height $\left(\mathrm{H}_{\mathrm{o}}\right)$ of the convex magnetic shaper on the axial deformation of the tube fitting. With the increase of $\mathrm{H}_{\mathrm{o}}$, the electromagnetic force subjected to the middle part of the tube fitting deforms from concave to convex, so the axial deformation also deforms from concave to convex, and there is an optimal $\mathrm{H}_{\mathrm{o}}$ $=34 \mathrm{~mm}$, which makes the axial deformation of the middle part of the tube fitting uniform. 


\subsection{Axial electromagnetic force and wall thickness of tube fittings}

This section studies the influence of different $\mathrm{H}_{i}$ and $\mathrm{H}_{0}$ on the distribution of axial electromagnetic force and the wall thickness at the center of tube fitting. The radial electromagnetic force subjected to tube fitting will promote axial deformation and lead to wall thickness thinning, while the axial electromagnetic force will increase the axial flow of tube fitting material and inhibit wall thickness thinning. In this paper, the relative wall thickness thinning index $R_{w}$ is introduced, which is defined as the wall thickness thinning divided by the bulging amount of tube fitting, and the wall thickness thinning is the absolute value of the outer wall displacement minus the inner wall displacement [17].In the analysis of this section, the midpoint displacement of the inner wall of the tube fitting is taken by the bulging measure, and the maximum value of the end of the tube fitting is taken by the axial electromagnetic force. Fig 10 and 11 show the influence of $\mathrm{H}_{\mathrm{i}}$ and $\mathrm{H}_{\mathrm{o}}$ on axial electromagnetic force and $\mathrm{R}_{\mathrm{w}}$ respectively. It can be seen that with the increase of $\mathrm{H}_{\mathrm{i}}$ and $\mathrm{H}_{\mathrm{o}}$ values, the axial electromagnetic force on tube fitting increases, which promotes the axial flow of tube fitting material and reduces the amount of wall thinning. Therefore, the axial electromagnetic force can be controlled by changing the length of the inner and outer wall of the convex shaper and the wall thinning can be restrained. When $\mathrm{H}_{\circ}$ is greater than the length of tube fitting, the axial electromagnetic force increases obviously. 


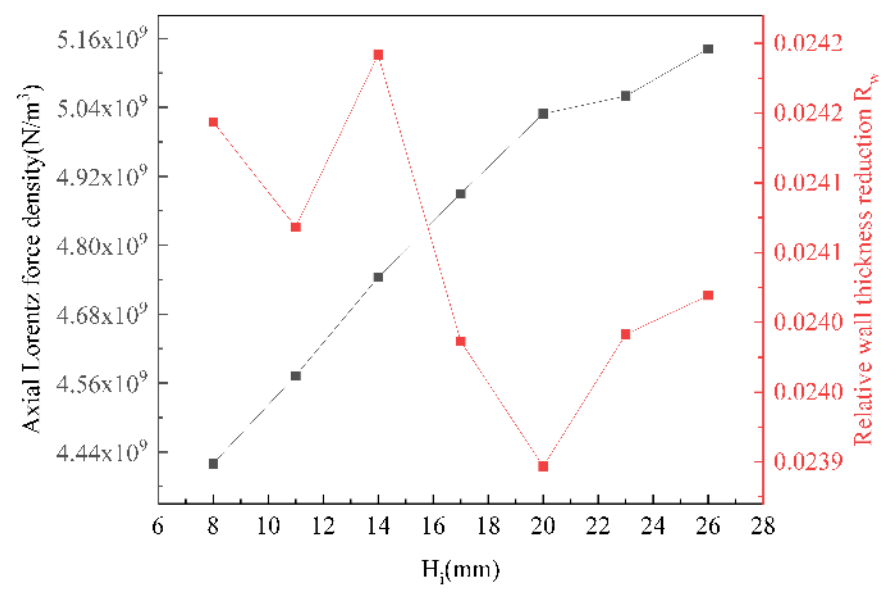

Fig.10 Influence of changing $\mathrm{H}_{\mathrm{i}}$ on axial electromagnetic force and $\mathrm{R}_{\mathrm{w}}$.

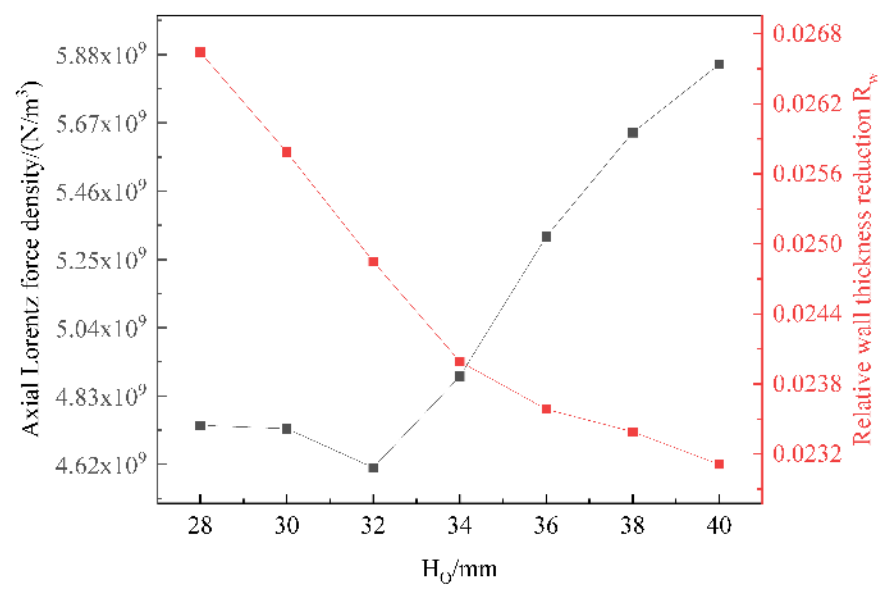

Fig.11 Influence of changing $\mathrm{H}_{\mathrm{o}}$ on axial electromagnetic force and $\mathrm{R}_{\mathrm{w}}$.

\section{Comparison and analysis}

This section will drive coil loading and traditional based on convex model of magnetic shaper load were analyzed, in order to make the analysis more accuracy, control parameters are the same, and two models fitting/biggest bulging mass were $10.21 \mathrm{~mm}$, the traditional model discharge voltage is $3.18 \mathrm{kV}$, based on the convex magnetic field shaper load down the electric voltage is $4.80 \mathrm{kV}$. The uniformity of axial deformation and wall thinning of tube fitting are analyzed from the Angle of radial and axial electromagnetic force. The main parameters of each material are shown in Tab 2. 
Table 2 Geometric parameters of drive coil and magnetic field shaper.

\begin{tabular}{|c|c|c|c|c|}
\hline Methods & Name & $\begin{array}{l}\text { Inner diameter } \\
\qquad(\mathrm{mm})\end{array}$ & $\begin{array}{l}\text { Outer diameter } \\
\qquad(\mathrm{mm})\end{array}$ & $\begin{array}{l}\text { Height } \\
(\mathrm{mm})\end{array}$ \\
\hline \multirow[t]{2}{*}{ Traditional } & Drive coil & 26.6 & 35.5 & 64.2 \\
\hline & Drive coil & 20.6 & 29.5 & 64.2 \\
\hline \multirow{2}{*}{$\begin{array}{l}\text { Based on } \\
\text { convex } \\
\text { magnetic } \\
\text { field shaper }\end{array}$} & $\begin{array}{c}\text { Convex } \\
\text { magnetic } \\
\text { field shaper } \\
\text { inner wall }\end{array}$ & 33.5 & - & 17 \\
\hline & $\begin{array}{c}\text { Convex } \\
\text { magnetic } \\
\text { field shaper } \\
\text { outer wall }\end{array}$ & - & 36.5 & 34 \\
\hline
\end{tabular}

Fig 12 (a) shows the comparison of radial electromagnetic force received by tube fittings under two loading modes. It can be seen that at $-25 \mathrm{~mm}-25 \mathrm{~mm}$, the radial electromagnetic force received by the tube fitting under the loading of convex magnetic field shaper is evenly distributed, while the distribution of electromagnetic force under the traditional loading mode is convex, and the peak value is located in the center of the tube fitting. Fig 12 (b) shows the comparison of axial electromagnetic force of tube fittings under two loading modes. At the end of the tube fitting, the loading scheme proposed in this paper, because the outer diameter height of the convex magnetic field shaper is larger than the tube fitting, provides the axial electromagnetic force density peak twice that of the traditional loading scheme, promotes the axial flow of the tube fitting material and restricts the wall thickness thinning. 


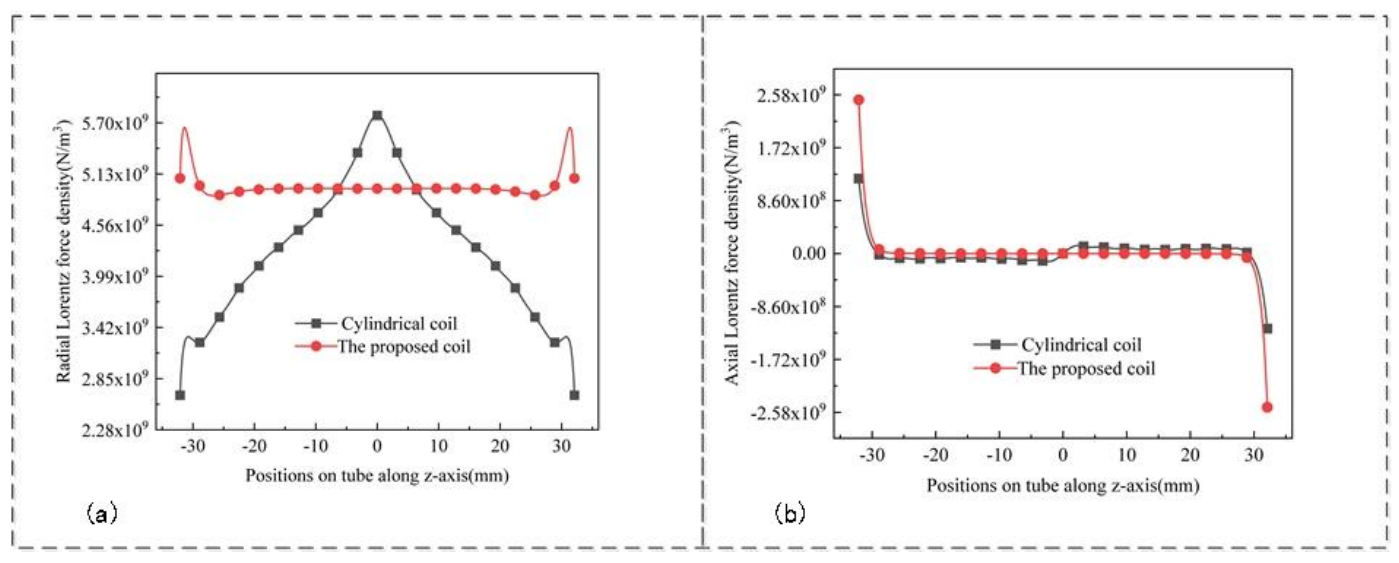

Fig.12 Electromagnetic force contrast (a) Radial; (b) Axial.

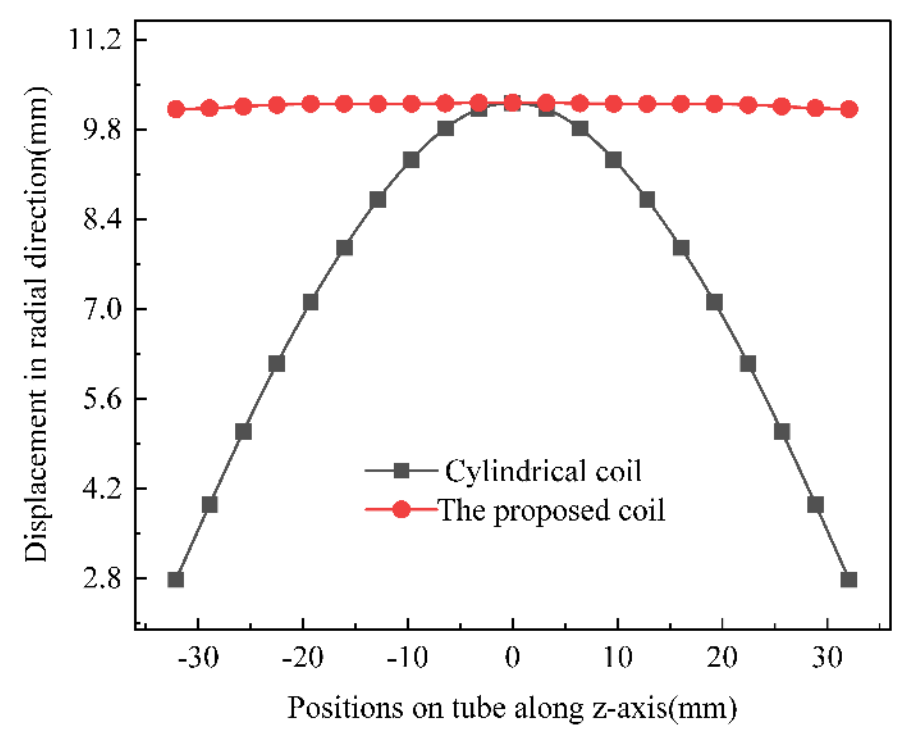

Fig.13 Comparison of axial deformation.

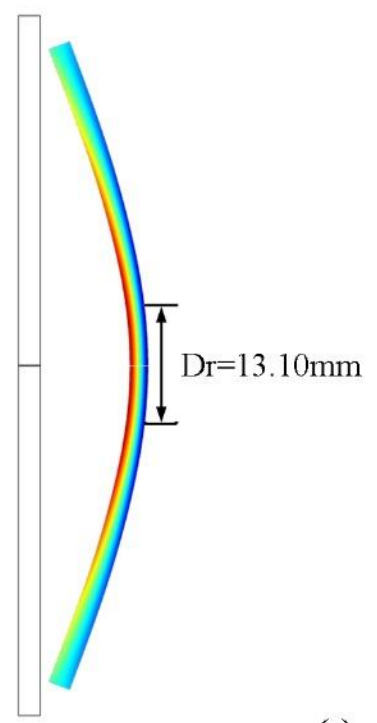

(a)

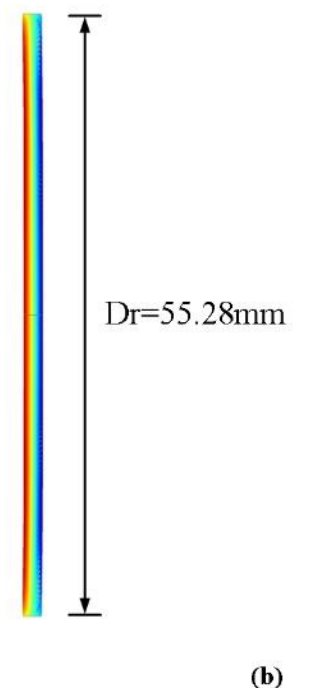


Fig.14 Bulging profile of tube fitting (a) Traditional loading mode; (b) Based on the loading mode of convex magnetic field shaper.

Fig 13 shows the comparison of tube fitting's axial deformation under two loading modes. In the traditional loading mode, due to the uneven distribution of electromagnetic force along the axial direction, the tube fitting expands into a convex shape with uneven axial direction, while in the loading mode based on the convex magnetic field shaper, the electromagnetic force is evenly distributed and the tube fitting's axial deformation is relatively smooth. The maximum deformation parameter $D_{r}$ is introduced, which is defined as the axial deformation length of tube fitting is less than or equal to $96 \%$ of the maximum deformation [14].As shown in Fig 14, $\mathrm{D}_{\mathrm{r}}=13.10$ $\mathrm{mm}$ when loading with traditional coil, while $\mathrm{D}_{\mathrm{r}}=55.28 \mathrm{~mm}$ when loading with the scheme proposed in this paper, which is the length of tube fittings finally formed. According to the calculation, the $R_{w}$ of traditional tube fittings is 0.03 , while the $R_{w}$ of the scheme proposed in this paper is 0.02 during loading, which increases the amount of wall thickness reduction by $33 \%$. Therefore, this loading method can inhibit wall thickness reduction.

\section{Theory conclusion}

In tube electromagnetic bulging axis to the problem of uneven deformation and wall thickness thinning, designed based on convex shaper load magnetic field of tube electromagnetic bulging system:

- Due to the convex magnetic current shaper lining and the outer wall current instead, tube fittings by concave radial electromagnetic force, and the central 
Analysis of Electromagnetic Force and Formability of Tube Electromagnetic Bulging Based on Convex Magnetic Field Shaper

tube fitting uniform radial electromagnetic force distribution, the axial deformation uniformity.

- When the external wall of convex magnetic shaper is higher than tube fitting, the axial electromagnetic force increases significantly, promoting the axial flow of tube material and restraining wall thinning.

- Compared with traditional tube electromagnetic bulging, the forming uniformity of tube fittings increases by 4.2 times, and the degree of wall thickness thinning decreases by $33 \%$. Experiments will be carried out in the future to verify the fitting between the model and simulation analysis. 


\section{Declarations}

\section{Funding}

This work was supported by the National Natural Science Foundation of China (NSFC) under Project Numbers 5187122 and 51707104.

\section{Availability of data and material}

All material data in this article are from previous work, the data is very reliable, and quoted in the article.

\section{Code availability}

These simulations are reliable and available and based on the finite element software COMSOL.

\section{Conflicts of interest}

The authors declare that they have no known competing financial interests or personal relationships that could have appeared to influence the work reported in this paper.

\section{Authors' contributions}

Weiye Wu: Methodology, Investigation, Writing -Original Draft, Writing

Li Qiu: Software, Methodology, Visualization

A. ABU-SIADA: Writing - Original Draft

Chenglin Wang: Formal analysis, Software

Jinbo Jiang: Conceptualization, Methodology 


\section{Reference}

[1] Chu Y Y, Lee R S, Psyk V, et al. Determination of the flow curve at high strain rates using electromagnetic punch stretching[J]. Journal of Materials Processing Tech, 2012, 212(6):1314-1323.

[2] Cao Q, Limeng D, Li Z, et al. Investigation of the Lorentz-force-driven sheet metal stamping process for cylindrical cup forming[J]. Journal of Materials Processing Technology, 2019.

[3] Qiu L, Wang C, Abu-Siada A, et al. Coil Temperature Rise and Workpiece Forming Efficiency of Electromagnetic Forming Based on Half-wave Current Method[J]. IEEE Access, 2020, PP(99):1-1.

[4] et al.Hai-ping, YU, and, et al. Tendency of homogeneous radial deformation during electromagnetic compression of aluminium tube[J]. Transactions of Nonferrous Metals Society of China, 2010.

[5] Li Z , Liu S , Li J , et al. Effect of coil length and relative position on electromagnetic tube bulging[J]. International Journal of Advanced Manufacturing Technology, 2018.

[6]Z. Jianet al., "Numerical simulation of electromagnetic progressive bulging and forming uniformity,'JJ. Plasticity Eng., vol. 19, no. 5, pp. 92-99, 2012.

[7] Qiu L, Yu Y, Xiong Q, et al. Analysis of Electromagnetic Force and Deformation Behavior in Electromagnetic Tube Expansion With Concave Coil Based on Finite Element Method[J]. IEEE Transactions on Applied Superconductivity, 2018, 28(3):1-5.

[8]Zhang, Xiao, Cao, et al. Radial Lorentz force augmented deep drawing for large drawing ratio using a novel dual-coil electromagnetic forming system[J]. Journal of Materials Processing Technology, 2015.

[9]Li F Q , Fang Y, Zhu Y, et al. Study on the homogeneity of deformation under electromagnetic expansion of metal tube[J]. International Journal of Applied Electromagnetics \& Mechanics, 2013, 42(1):13-25.

[10]Qiu L , Cao Q , Zhang W , et al. Electromagnetic Force Distribution and Wall Thickness Reduction of Three-Coil Electromagnetic Tube Bulging With Axial Compression[J]. IEEE Access, 2020, 8:1-1.

[11]Bahmani M A , Niayesh K , Karimi A . 3D Simulation of magnetic field distribution in electromagnetic forming systems with field-shaper[J]. Journal of Materials Processing Technology, 2009, 209(5):2295-2301.

[12]Huang Hao. Research on shape correction of sheet metal electromagnetic forming based on magnetic collector [D]. Three Gorges University

[13]Qiu L , Li Y , Yu Y, et al. Electromagnetic Force Distribution and Deformation Homogeneity of Electromagnetic Tube Expansion With a New Concave Coil Structure[J]. IEEE Access, 2019, PP(99):1-1.

[14]Paese E, Rosa P A R , Geier M, et al. An Analysis of Electromagnetic Sheet Metal Forming Process[J]. Applied Mechanics \& Materials.

[15] Shang J, Wilkerson L, Hatkevich S. Hemming of Aluminum Alloy Sheets Using Electromagnetic Forming[J]. Journal of Materials Engineering \& Performance, 2011, 20(8):1370.

[16]Qiu L, Wang C, Abu-Siad A, et al. Parametric Simulation Analysis of the 
Analysis of Electromagnetic Force and Formability of Tube Electromagnetic Bulging Based on Convex Magnetic Field Shaper

Electromagnetic Force Distribution and Formability of Tube Electromagnetic Bulging Based on Auxiliary Coil[J]. IEEE Access, 2020, PP (99):1-1. 


\section{Figure Captions}

Fig.1 Electromagnetic bulging structure diagram (a) traditional model; (b) loading model based on convex magnetic field shaper.

Fig.2 Electromagnetic bulging diagram (a) Simulation; (b) Experiment [14].

Fig.3 Geometrical structure diagram (a) Bulging of traditional tube fittings; (b) Bulging of tube fittings loaded by convex magnetic field shaper.

Fig.4 Bulging simulation flow chart of tube fittings.

Fig.5 Electromagnetic bulging equivalent circuit of tube fitting based on convex magnetic field shaper.

Fig.6 Change the radial electromagnetic force distribution of $\mathrm{H}_{\mathrm{i}}$.

Fig.7 Change $\mathrm{H}_{\mathrm{i}}$ tube fitting axial deformation.

Fig.8 Change the radial electromagnetic force distribution of $\mathrm{H}_{\mathrm{o}}$.

Fig.9 Change $\mathrm{H}_{\mathrm{o}}$ tube fitting axial deformation.

Fig.10 Influence of changing $\mathrm{H}_{\mathrm{i}}$ on axial electromagnetic force and $\mathrm{R}_{\mathrm{w}}$.

Fig.11 Influence of changing $\mathrm{H}_{\mathrm{o}}$ on axial electromagnetic force and $\mathrm{R}_{\mathrm{w}}$.

Fig.12 Electromagnetic force contrast (a) Radial; (b) Axial.

Fig.13 Comparison of axial deformation.

Fig.14 Bulging profile of tube fitting (a) Traditional loading mode ;(b) Based on the loading mode of convex magnetic field shaper. 